\title{
Characterization of low molecular weight urinary proteins at varying time intervals in type 2 diabetes mellitus and diabetic nephropathy patients
}

\author{
Dhara N. Patel ${ }^{1}$ and Kiran Kalia ${ }^{2^{*}}$
}

\begin{abstract}
Background: To identify low molecular weight urinary proteins capable of detecting diabetic nephropathy patients which may predict renal alterations at early stages and prevent it from worsening further.

Method: Three hundred ninety (390) age-matched subjects were divided into 8 groups depending upon duration of diabetes and the severity of renal damage. Urinary proteome profile of all subjects was determined with the help of microfluidic array. Participants with similar profile were further selected to study proteome map of urinary low molecular weight proteins with the help of 2 dimensional gel electrophoresis.

Results: Out of 390 total patients 268 patients showed a similar one dimensional proteomic pattern. Further, twodimensional urinary proteomic pattern of these patients with molecular weight $<50 \mathrm{kDa}$ was studied. Eight proteins with molecular weight 11, 15, 17, 23, 34, 38 and $46 \mathrm{kDa}$ were identified with MALDI-QTOF. These low molecular weight proteins showed gradual increase in urinary excretion along with the duration of diabetes and severity of renal damage.

Conclusion: The study concludes that proteomic analysis might be a useful tool for detecting some novel markers capable of detecting patients susceptible to diabetic nephropathy in the early phase.
\end{abstract}

Keywords: Urinary 2DE, Urinary proteomics, Diabetic nephropathy, Diabetic kidney disease

\section{Introduction}

Type 2 diabetes mellitus (T2DM) patients are frequently diagnosed with well established microalbuminuric stage due to its inadequate diagnosis and prognosis [1]. The progression of diabetic nephropathy $(\mathrm{DN})$, generally silent and remains clinically unnoticed [2]. DN, if undiagnosed or untreated leads to end-stage renal disease and the patients are solely left with only two survival options which are costly, dialysis or kidney transplantation. The morbidity and mortality rate of DN patients are escalating and there is thus a urgent need for new promising

\footnotetext{
*Correspondence: kirankalia@gmail.com; director@niperahm.ac.in 2 Present Address: National Institute of Pharmaceutical Education and Research (NIPER-G), Gandhinagar Opposite Air Force Station, Palaj, Gandhinagar, Gujarat 382355, India

Full list of author information is available at the end of the article
}

biomarkers capable of diagnosing DN prior to appearance of microalbumin and rise in serum creatinine. This may also lead to suggesting some new strategies for therapeutics.

Urinary proteomics has recently been applied to understand pathophysiology and complexity of pathogenic mechanisms during T2DM induced nephropathy $[3,4]$. The proteomic techniques are considered sensitive capable of detecting low abundance proteins. We compared two-dimensional urinary proteomic pattern of proteins with molecular weight $<50 \mathrm{kDa}$. For detecting the earliest possible LMWP, T2DM patients without any secondary complications (T2DM duration 1-20 years), and T2DM patients with microalbuminuria and nephropathy as a secondary complication were included. Further, the pattern obtained from T2DM 
patients with and without nephropathy was compared with protein pattern obtained from non-diabeticnephropathies (NDN) like IgA nephropathy, Focal Segmental Glomerulosclerosis, Minimal Change Disease and Nephrotic Syndrome patients. We assumed that while comparing protein profiles of all study groups, it may be useful in selecting LMWPs that are exclusive for T2DM only. The present study thus was designed to isolate and identify urinary low abundant and low molecular weight proteins (LMWPs) $(<50 \mathrm{kDa})$ which can predict the possibility of diabetic nephropathy at earliest. It will further be helpful in the pathophysiological and therapeutic interventions for diabetes and diabetic nephropathy.

\section{Methodology}

Patients attending outpatient's clinic of Muljibhai Patel Urological Hospital, Nadiad, Gujarat, India, between December 2009 and April 2013 were enrolled for the present cross-sectional study. The study plan was approved by the institution's Ethical Committee. All patients fulfilled; inclusion criteria i.e. age $>25$ and estimated GFR $\left(\right.$ eGFR) $>30 \mathrm{ml} / \mathrm{min} / 1.73 \mathrm{~m}^{2}$. Patients with any disorders/diseases, undergone any surgery, past history of consuming any drugs, lactating females and pregnant women were excluded. All T2DM patients were either on insulin, oral hypoglycemic agents (OHA) like metformin/glibenclamide or a combination of both. Each patient provided fasting blood/urine samples. The blood samples were collected in plain as well as EDTA coated pre-sterile vacutainers (BD-Biosciences purchased). Blood was centrifuged at $3000 \mathrm{rpm}$ at $4{ }^{\circ} \mathrm{C}$ for the collection of serum/plasma and stored at $-80{ }^{\circ} \mathrm{C}$ for further analysis. Clean midstream urine samples were collected in pre-sterile containers (Tarson) and centrifuged at $3000 \mathrm{rpm}$ for $15 \mathrm{~min}$ at $4{ }^{\circ} \mathrm{C}$ to remove the cellular debris. The aliquots were stored at $-80{ }^{\circ} \mathrm{C}$ with protease inhibitors to prevent further protein degradation. Participants were divided into various groups based on the duration of diabetes:

Group I: normal healthy adults (NHA); Group II: T2DM participants with duration of $0-5$ years; Group III: T2DM participants with duration of 5-10 years; Group IV: T2DM participants with duration of 10-15 years; Group V: T2DM participants with duration of 15-20 years; Group VI: microalbuminuria with T2DM duration of $>15$ years (MIA); Group VII: diabetic nephropathy with T2DM duration of $>15$ years (DN); Group VIII: non-diabetic nephropathy (NDN) which includes; IgA nephropathy $(\mathrm{N}=22)$, focal segmental glomerulosclerosis $(\mathrm{N}=12)$, minimal change disease $(\mathrm{N}=9)$, nephrotic syndrome $(\mathrm{N}=7)$.

\section{Biochemical assay}

Serum and urinary creatinine levels were measured by Jaffe's Kinetic method [5]. Glomerular filtration rate (eGFR) was calculated by Cockroft and Gaults Equation [6]. The Glycated Hemoglobin was quantified by the method of Parker et al. [7]. Fasting blood glucose was estimated based on GOD-POD enzymatic reaction using Automatic Random Access Biochemistry Analyzer XL-300 (Erba Diagnostics, Mannheim). Urinary microalbumin was determined using ELISA-based diagnostic method (Biovendor Research Diagnostics; European Union).

\section{Microfluidic chip array and 1 dimensional electrophoresis} Urinary total protein was precipitated by acetone method. To $5 \mathrm{ml}$ of the urine sample, $40 \%$ ice-cold acetone was added slowly and gradually. The urine-solvent mixture was kept at $-20{ }^{\circ} \mathrm{C}$ for $4 \mathrm{~h}$ following centrifugation at $14,000 \times g$ for $20 \mathrm{~min}$ to obtain the precipitated protein. The total protein obtained was further quantified by the Bicinchoninic acid method following the instructions manual of QuantiProTM BCA Assay Kit provided by Sigma. The automated capillary electrophoresis was carried out by following instructions according to Protein 230 Kit supplied by Agilent Life Technologies. The assay was performed on Agilent 2100 Bioanalyzer. The sensitivity and quantitative detection limit of the assay are $30-2000 \mathrm{ng} / \mu \mathrm{l}$ protein.

\section{Sample preparation for 2-dimensional gel electrophoresis (2DE)}

Five patients with similar urinary 1 dimensional protein profile from each group were selected for further $2 \mathrm{DE}$ study. The 2DE of each selected participants were carried out twice. Low molecular weight proteins (LMWPs) from precipitated proteins were isolated with the help of centrifugal cutoff membranes procured from Merck Millipore. For obtaining LMWPs up to $50 \mathrm{kDa}, 100 \mathrm{kDa}$ cutoff followed by a $3 \mathrm{kDa}$ cutoff was applied. The procedure was according to instructions manual of Merck, and the centrifugation was done at $7500 \times g$ for $45 \mathrm{~min}$ at $4{ }^{\circ} \mathrm{C}$. For collecting the protein samples from the cutoff membrane, an inverse spin centrifugal technique was applied. The protein obtained was then dissolved in the rehydration buffer and quantified using BCA method.

\section{2-Dimensional gel electrophoresis}

For isoelectric focusing an immobilized $\mathrm{pH}$ gradient (IPG) strip of $\mathrm{pH} 3$ to 10 and $7 \mathrm{~cm}$ in length (BioRad) was loaded with $150 \mu \mathrm{g}$ protein. The gel strip was rehydrated along with the sample for $14 \mathrm{~h}$ at $20^{\circ} \mathrm{C}$. The samples were 
focused for $5 \mathrm{~h}$ up to $10,000-\mathrm{V}$ h. The IPG strip was then equilibrated with dithiothreitol equilibration buffer and iodoacetamide equilibration buffer for $15 \mathrm{~min}$ each.

\section{Second dimension 2D-PAGE}

The second dimension was carried out on $8-16 \%$ SDSpolyacrylamide pre-casted Tricine gels procured from BioRad. The run was conducted at $10 \mathrm{mAmp}$ for $8 \mathrm{~h}$ maintaining $4{ }^{\circ} \mathrm{C}$. A low molecular weight marker was run along with the samples.

\section{Coomassie blue staining and image analysis}

The gels were rinsed with deionized water for $1 \mathrm{~min}$ with constant shaking. Further, the gels were transferred to Coomassie blue stain. The gel was left in the staining solution for overnight on the shaker. Next day the gel was transferred to the detaining solution. The gel was destained till the background was clear. Once the gel was destained, it was further analyzed with the image analyzer (BioRad). The image was captured, and the gel spots were compared using Lab Image software (BioRad). The protein spots were then manually cut and stored in deionized water and sent for Q-TOF analysis.

\section{Protein identification by Q-TOF analysis}

Spots isolated from the gel were destained and digested in-gel with trypsin for $24 \mathrm{~h}$ at $37{ }^{\circ} \mathrm{C}$. Tryptic peptides were extracted with $0.1 \%$ trifluoroacetic acid, purified using Zip-TipC18 pipette tips from Millipore, and analyzed on a 6550 I-Funnel quadrupole time-of-flight mass spectrometer (Q-TOF LC/MS) (Agilent Technologies), connected to a CapLC. An MS-MS survey method was used to acquire MS-MS spectra. Data analysis was performed using the Data Processing Software-Agilent Technologies Spectrum Mill MS Proteomics Workbench (Rev B.04.00.127). Peptide alignment was performed using peptide alignment tool of Mass Hunter, Mill Spectrum (Agilent) connected to Swiss-Prot database. Peptides consisting of five or more amino acids were used and matched to either a non-redundant human IPI or the Swiss-Prot database to identify the corresponding proteins.

\section{Statistical analysis}

Comparisons among groups were performed using ANOVA with Tukey's posthoc test for multiple comparisons, $\mathrm{p}$ values $<0.05$ were considered statistically significant. The data are reported as mean $\pm \mathrm{SD}$. For analyzing one-dimensional profile, the differences in frequency of all proteins (spectral data) were arbitrarily determined. The presence of a protein was greater than $80 \%$ in one group, but less than $20 \%$ in the other group was considered significant. The coefficient of variation $(\mathrm{CV})$ was calculated using the formula: \%CV) standard deviation/ mean $\times 100 \%$.

\section{Results}

Anthropometric and clinical data of patients

Table 1 summarizes the details of anthropometric parameters, serum and urinary biochemical parameters of 390 subjects. The participants were distributed in eight different groups on the basis of their duration of diabetes and renal function. Body Mass Index (BMI) increased moderately $(\mathrm{p}=0.126)$ in all the test groups compared to healthy controls. All T2DM patients, with or without nephropathy showed significantly increased fasting blood glucose and glycated hemoglobin in comparison to healthy controls $(\mathrm{p}<0.001)$. Significantly increased serum creatinine and microalbumin levels were observed only in MIA, DN and NDN patients $(\mathrm{p}<0.001)$. Further, a significant decline in eGFR of patients with MIA, DN, and NDN $(p<0.001)$ was observed. However, microalbumin, serum creatinine, and eGFR were within normal range in T2DM patients with $0-5,5-10,10-15$ and $15-20$ years of diabetes duration.

\section{One dimensional urinary proteomic profile of T2DM patients}

Proteins from every single urine samples were differentiated in the form of bands depending on their molecular weight with the help of microfluidic array. The protein profile was in the form of one-dimensional SDS-PAGE electropherogram (Fig. 1a-h) (Additional file 1). These data provided a detailed description of concentration and molecular weight of individual fractionated protein. A single protein was detected with its corresponding spectral peak and particular elution time. The peaks of the proteins/peptides were studied for identifying whether the proteins are single or a mixture of protein. A crisp and sharp peak indicated the presence of one single protein/peptide. While a blunt and broad peak indicated the presence of two or mixture of proteins/peptides. Such overlapping peaks were observed due to proteins of similar molecular weight. Thus, such peaks were not considered for further analysis. The selection of candidate proteins was based upon its frequency of appearance. Only those proteins with frequency more than $80 \%$ in group I, II, III, IV, V, VI, VII and less than $20 \%$ in group VIII were selected. This selection was to assure that the candidate proteins are specific for nephropathy due to T2DM as a triggering agent. Which implies to the fact that the proteins considered for further analysis were those thus, the protein bands obtained due to sharp peaks were further considered for the study. Further, maximum patients showing a similar protein profile were 
Table 1 Fasting glucose, serum fructosamine, HbA1C, serum and urinary creatinine, eGFR, urinary microalbumin and total protein in control and test groups

\begin{tabular}{|c|c|c|c|c|c|c|c|c|}
\hline \multirow{2}{*}{$\begin{array}{l}\text { Serum } \\
\text { and urinary } \\
\text { biochemical } \\
\text { parameters }\end{array}$} & \multirow[t]{2}{*}{ Control } & \multicolumn{4}{|c|}{ Type 2 diabetes (T2DM) with normoalbuminuria } & \multirow{2}{*}{$\begin{array}{l}\text { Type } 2 \text { diabetes } \\
\text { with micro } \\
\text { albuminuria }\end{array}$} & \multirow{2}{*}{$\begin{array}{l}\text { Diabetic } \\
\text { nephropathy }\end{array}$} & \multirow{2}{*}{$\begin{array}{l}\text { Non diabetic } \\
\text { nephropathy }\end{array}$} \\
\hline & & $0-5$ years & $5-10$ years & $10-15$ years & $15-20$ years & & & \\
\hline No. of patients & 48 & 59 & 38 & 37 & 25 & 50 & 83 & 50 \\
\hline BMI $\left(\mathrm{Kg} / \mathrm{m}^{2}\right)$ & $21.57 \pm 0.9$ & $\begin{array}{l}27.21 \pm 0.7 \\
\mathrm{NS}_{\mathrm{a}}\end{array}$ & $\begin{array}{l}26.52 \pm 0.7 \\
\mathrm{NS} a b\end{array}$ & $\begin{array}{l}26.21 \pm 0.3 \\
\text { Nsabc }\end{array}$ & $\begin{array}{l}26.11 \pm 0.5 \\
\text { Nsabcd }\end{array}$ & $\begin{array}{l}23.92 \pm 0.7 \\
\text { Nsabcde }\end{array}$ & $\begin{array}{l}24.01 \pm 0.4 \\
\text { Nsabcdef }\end{array}$ & $\begin{array}{l}23.62 \pm 0.8 \\
\text { Nsabcdefg }\end{array}$ \\
\hline $\begin{array}{l}\text { Fasting glucose } \\
\mathrm{mg} / \mathrm{dl}\end{array}$ & $93.96 \pm 2.1$ & $\begin{array}{l}149.4 \pm 7.5 \\
* *{ }_{a}\end{array}$ & $\begin{array}{l}138.5 \pm 7.5 \\
{ }_{* * *} \mathrm{a}^{\mathrm{NS} b}\end{array}$ & $\begin{array}{l}147.4 \pm 5.3 \\
* * a^{*}{ }^{N} \mathrm{bc}\end{array}$ & $\begin{array}{l}150.2 \pm 6.7 \\
* * * a \mathrm{NS} \text { bed }\end{array}$ & $\begin{array}{l}155.6 \pm 4.2 \\
* * \text { a a S bcde }^{*}\end{array}$ & $\begin{array}{l}157.6 \pm 5.5 \\
* * * a^{N S} \text { bcdef }\end{array}$ & $\begin{array}{l}92.8 \pm 3.8 \\
\mathrm{NS}_{\mathrm{a}}\end{array}$ \\
\hline $\mathrm{HbA} 1 \mathrm{C} \%$ & $4.5 \pm 0.3$ & $\begin{array}{l}7.5 \pm 1.3 \\
* * * a\end{array}$ & $\begin{array}{l}7.0 \pm 0.2 \\
* * * \mathrm{a}^{\mathrm{NS}} \mathrm{b}\end{array}$ & $\begin{array}{l}8.6 \pm 0.3 \\
{ }_{* * *} \mathrm{~N}^{\mathrm{NS}} \mathrm{bc}\end{array}$ & $\begin{array}{l}8.9 \pm 0.5 \\
* * a^{N S} b c d\end{array}$ & $\begin{array}{l}9.6 \pm 0.8 \\
{ }_{* * *} \mathrm{a}^{\mathrm{NS}} \mathrm{bcde}\end{array}$ & $\begin{array}{l}10.6 \pm 0.8 \\
* * * a^{N S} \text { bcdef }\end{array}$ & $\begin{array}{l}4.3 \pm 0.1 \\
\mathrm{NS}_{\mathrm{a}}\end{array}$ \\
\hline $\begin{array}{l}\text { Serum fructosa- } \\
\text { mine }\end{array}$ & $143.3 \pm 7.4$ & $\begin{array}{l}300.6 \pm 19.0 \\
* * * a\end{array}$ & $\begin{array}{l}315.6 \pm 13.8 \\
* * * a b^{N S}\end{array}$ & $\begin{array}{l}354.2 \pm 18.6 \\
* * * a^{\mathrm{NS}} \mathrm{bc}\end{array}$ & $\begin{array}{l}428.8 \pm 27.5 \\
* * * a * b c^{* 5} d\end{array}$ & $\begin{array}{l}469.4 \pm 17.5 \\
* * * a b c d^{N S} \mathrm{e}\end{array}$ & $\begin{array}{l}523.5 \pm 12.4 \\
{ }^{* * *} a b c^{* *} d^{\text {NS }} \text { ef }\end{array}$ & $\begin{array}{l}158.4 \pm 8.06 \\
\mathrm{NS}_{\mathrm{a}}\end{array}$ \\
\hline $\begin{array}{l}\text { Serum creatinine } \\
\mathrm{mg} / \mathrm{dl}\end{array}$ & $0.75 \pm 0.1$ & $\begin{array}{l}0.89 \\
\mathrm{NS}_{\mathrm{a}}\end{array}$ & $\begin{array}{l}0.9 \pm 0.04 \\
{ }_{\mathrm{NS}} \mathrm{ab}\end{array}$ & $\begin{array}{l}1.1 \pm 0.7 \\
\mathrm{NS}_{\mathrm{abc}}\end{array}$ & $\begin{array}{l}1.1 \pm 0.1 \\
\text { Ns abcd }\end{array}$ & $\begin{array}{l}1.7 \pm 0.7 \\
* * * a b c^{N S} \text { de }\end{array}$ & $\begin{array}{l}4.3 \pm 0.8 \\
* * * a b c d e f\end{array}$ & $\begin{array}{l}4.9 \pm 0.2 \\
* * * \text { abcdef }{ }^{\mathrm{NS}} \mathrm{g}\end{array}$ \\
\hline $\begin{array}{l}\text { eGFR ml/ } \\
\mathrm{min} / 1.73 \mathrm{~m}^{2}\end{array}$ & $94.7 \pm 4.3$ & $\begin{array}{l}98.8 \pm 4.7 \\
\mathrm{NS}_{\mathrm{a}}\end{array}$ & $\begin{array}{l}98.2 \pm 5.0 \\
{ }^{\mathrm{NS}} \mathrm{ab}\end{array}$ & $\begin{array}{l}96.3 \pm 6.4 \\
{ }^{N 5} a b c\end{array}$ & $\begin{array}{l}80.0 \pm 6.1 \\
\text { NSabcd }\end{array}$ & $\begin{array}{l}63.3 \pm 5.3 \\
* * * a b c d{ }^{* *} \mathrm{e}\end{array}$ & $\begin{array}{l}34.5 \pm 1.9 \\
{ }^{* * *} \mathrm{abcd}{ }^{* *} e^{* f}\end{array}$ & $\begin{array}{l}33.4 \pm 4.4 \\
* * * \\
\text { abcde }\end{array}$ \\
\hline $\begin{array}{l}\text { Urinary micro } \\
\text { albumin mg/g } \\
\text { creatinine }\end{array}$ & $12.1 \pm 0.7$ & $\begin{array}{l}12.6 \pm 0.9 \\
\mathrm{NS}_{\mathrm{a}}\end{array}$ & $\begin{array}{l}10.6 \pm 1.1 \\
{ }_{\mathrm{NS}} \mathrm{ab}\end{array}$ & $\begin{array}{l}12.45 \pm 3.1 \\
\text { Ns abc }\end{array}$ & $\begin{array}{l}12.73 \pm 1.9 \\
\text { Nsabcd }\end{array}$ & $\begin{array}{l}165.1 \pm 10.2 \\
* * * a b c d e\end{array}$ & $\begin{array}{l}322.5 \pm 7.8 \\
* * * a b c d e f\end{array}$ & $\begin{array}{l}329.8 \pm 4.5 \\
* * * \\
\text { abcdef }\end{array}$ \\
\hline $\begin{array}{l}\text { Urinary total pro- } \\
\text { tein by BCA mg/ } \\
\text { dl urine }\end{array}$ & $9.6 \pm 0.78$ & $\begin{array}{l}10.7 \pm 0.56 \\
\mathrm{NS}_{\mathrm{a}}\end{array}$ & $\begin{array}{l}17.8 \pm 0.1 \\
\mathrm{NS} a \mathrm{ab}\end{array}$ & $\begin{array}{l}18.1 \pm 0.34 \\
\text { NSabc }\end{array}$ & $\begin{array}{l}19.4 \pm 0.11 \\
\text { Nsabcd }\end{array}$ & $\begin{array}{l}186.4 \pm 0.96 \\
* * * a b c d e\end{array}$ & $\begin{array}{l}448.3 \pm 0.12 \\
* * * a b c d e f\end{array}$ & $\begin{array}{l}450.1 \pm 0.89 \\
{ }^{* *} \text { abcdef }{ }^{\mathrm{NS}} \mathrm{g}\end{array}$ \\
\hline $\begin{array}{l}\text { Urinary total } \\
\text { protein by micro- } \\
\text { fluidic array mg/ } \\
\text { dl urine }\end{array}$ & $13.7 \pm 0.5$ & $\begin{array}{l}13.8 \pm 0.7 \\
\mathrm{NS}_{\mathrm{a}}\end{array}$ & $\begin{array}{l}21.3 \pm 0.1 \\
\mathrm{NS}_{\mathrm{ab}}\end{array}$ & $\begin{array}{l}23.4 \pm 0.18 \\
\text { NSabc }\end{array}$ & $\begin{array}{l}26.9 \pm 0.27 \\
\text { Ns abcd }\end{array}$ & $\begin{array}{l}192.4 \pm 1.6 \\
* * * a b c d e f\end{array}$ & $\begin{array}{l}568.9 \pm 0.57 \\
* * * a b c d e f\end{array}$ & $\begin{array}{l}571.8 \pm 0.46 \\
* * * a b c d e f^{N S} g\end{array}$ \\
\hline
\end{tabular}

The results are expressed as mean $+\mathrm{SE}$ and $\mathrm{p}<0.05$ is considered significant. ${ }^{*} \mathrm{p}<0.05,{ }^{* *} \mathrm{p}<0.001,{ }^{* * *} \mathrm{p}<0.0001$

a-compared with control, b-compared with 0-5 years T2DM, c-compared with 5-10 years T2DM, d-compared with 1-15 years T2DM, e-compared with 15-20 years T2DM, f-compared with microalbuminuria, g-compared with diabetic nephropathy

NS, non significant

selected for further analysis. Patients with different protein excretion pattern and less in number were not considered (Fig. 1a-h).

Out of 48, only 35 normal healthy adults (NHA) showed a similar urinary protein pattern. Further, total 159 T2DM patients without any secondary complications were distributed into four different groups depending upon the duration of diabetes. Wherein, 37 out of 59 patients with $0-5$ years T2DM duration showed protein pattern analogous to that of the NHA group. We observed that proteins of molecular weight 13, 17, 23, $24,28,35,37,39,41,49,50 \mathrm{kDa}$ were found significant in rest of the T2DM (Table 2) groups. Further, these proteins were found to increase gradually in 33 out of 38 patients of T2DM with 5-10 years of duration, 20 out of 37 patients with 10-15 years of T2DM and 19 out of 25 T2DM patients with 15-20 years of diabetes duration (Table 2) $(\mathrm{p}<0.001) .39$ out of 50 MIA patients and 69 out of $83 \mathrm{DN}$ patients also showed a significant increase in the above-mentioned proteins (Table 2) $(\mathrm{p}<0.001)$. 16 out of 50 patients from NDN group showed non-significant excretion of the LMWPs found in other T2DM groups (Table 2). However, NDN showed either lower or no concentration of the respective proteins (data of 260 one dimensional urinary profile is provided in Additional file 1). Thus, patients with such low molecular weight proteinuria were further selected for 2-dimensional electrophoresis study.

\section{Identification of isolated proteins by 2D-PAGE}

The study predicted a significant set of proteins that are differentially expressed in the urine of T2DM patients with varying duration of diabetes (ranging from 1 to 20 years), microalbuminuria and diabetic nephropathy. As shown in Fig. 2a-f, total 25 areas from the 2-D gels representing single or multiple spots corresponding to the same protein were picked for further analysis. Analysis of MS/MS spectra by MS Spectrum Mill, de novo sequencing algorithms and peptide mass fingerprinting by MASCOT Analysis was carried on. This analysis includes mass by charge ratio and the probability based on MOWSE score (i.e. Molecular Weight SEarch) which helps to identify the protein. Peptides with the MOWSE score $>85$ were selected, and 186 peptides were further 
a Profile of Control Group

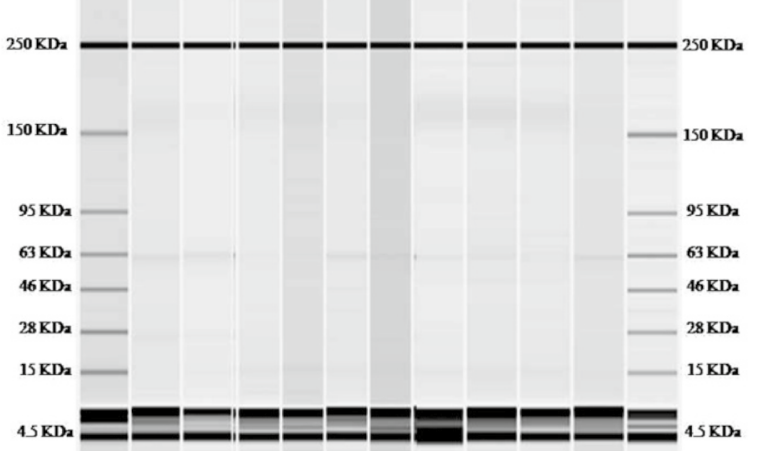

c Profile of 5-10 yrs T2DM Group

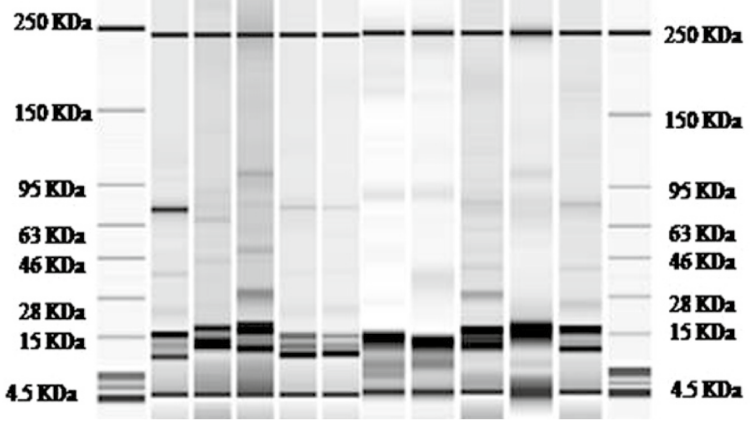

e Profile of 15-20 yrs T2DM Group

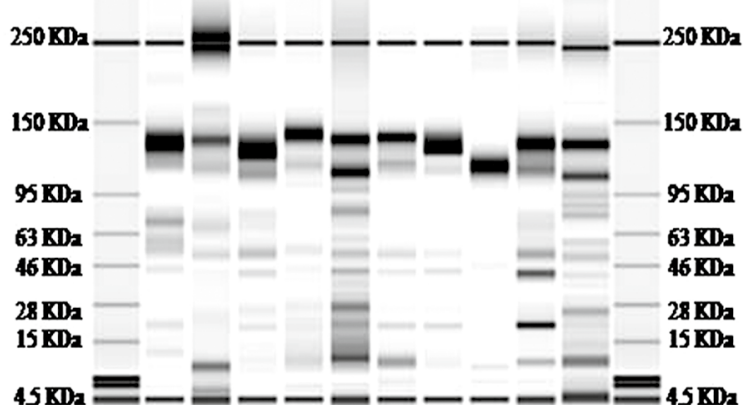

g Profile of Diabetic Nephropathy Group

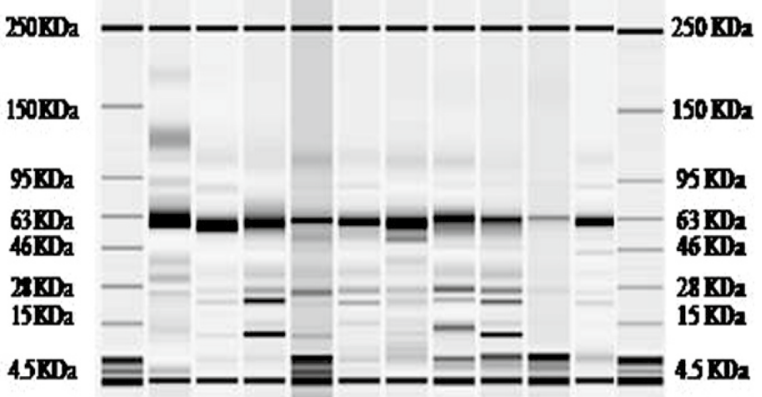

b Profile of 0 -5 yrs T2DM Group

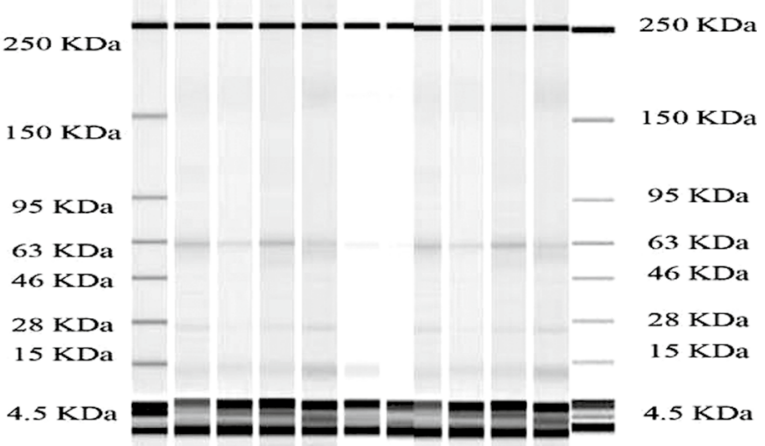

d Profile of 10-15 yrs T2DM Group

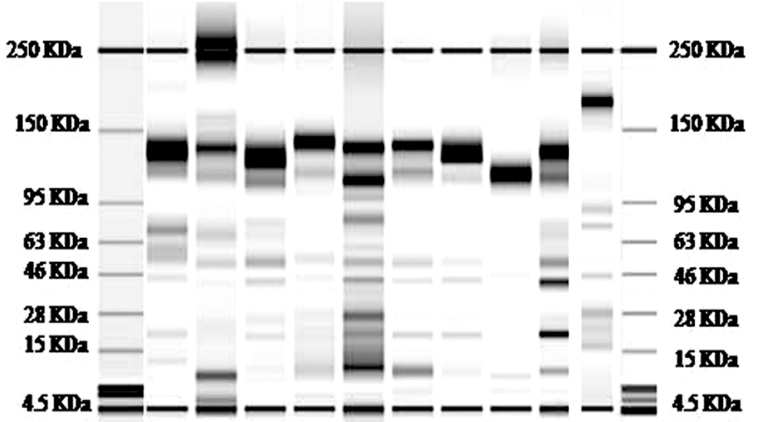

f Profile of Microalbuminuria Group

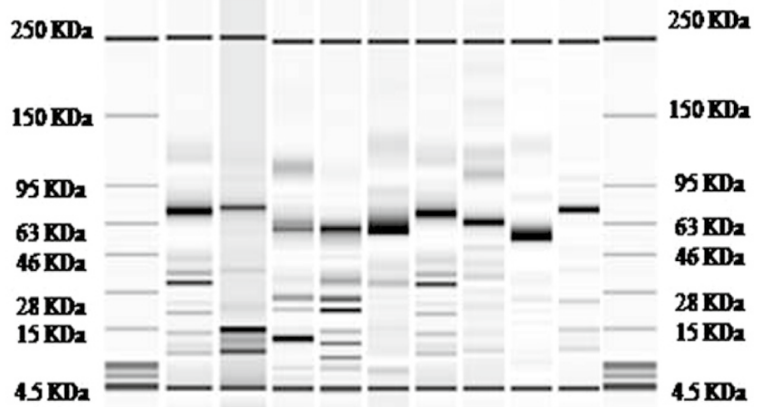

h Profile of Non Diabetic Nephropathy Group

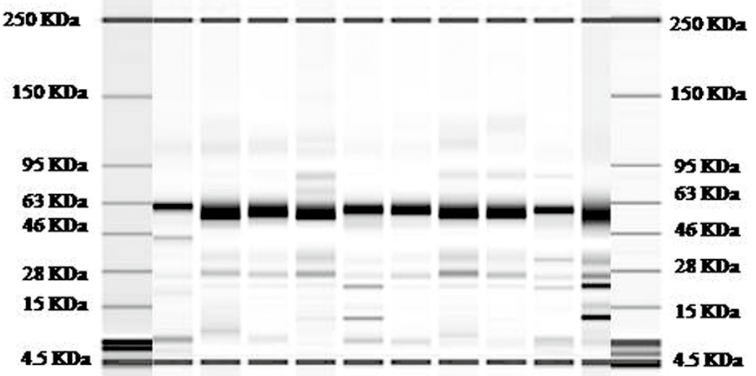

Fig. 1 Urinary protein profile of various groups. One dimensional protein profile of 390 participants showing various range of proteins excreted in urine. Total 268 participants showed a similar profile (Additional file 1) and were further selected for 2 dimensional gel electrophoresis study. The present one dimensional profile depicted appearance of low molecular weight proteinuria of clinical significance in early diagnosis of diabetic nephropathy 
Table 2 Concentration of low molecular weight urinary proteins in T2DM patients with and without secondary complications

\begin{tabular}{|c|c|c|c|c|c|c|c|c|}
\hline $\begin{array}{l}\text { Molecular } \\
\text { weight in kDa }\end{array}$ & Control (mg/dl) & $\begin{array}{l}\text { T2DM with 0-5 } \\
\text { years }(\mathrm{mg} / \mathrm{dl})\end{array}$ & $\begin{array}{l}\text { T2DM with 5-10 } \\
\text { years (mg/dl) }\end{array}$ & $\begin{array}{l}\text { T2DM } \\
\text { with } 10-15 \text { years } \\
\text { (mg/dl) }\end{array}$ & $\begin{array}{l}\text { T2DM } \\
\text { with } 15-20 \\
\text { years }(\mathrm{mg} / \mathrm{dl})\end{array}$ & $\begin{array}{l}\text { Micro } \\
\text { albuminuria } \\
(\mathrm{mg} / \mathrm{dl})\end{array}$ & $\begin{array}{l}\text { Diabetic } \\
\text { nephropathy } \\
\text { (mg/dl) }\end{array}$ & $\begin{array}{l}\text { Non diabetic } \\
\text { nephropathy } \\
\text { (mg/dl) }\end{array}$ \\
\hline 13 & 1.5 & $\begin{array}{l}3.01 \\
\mathrm{NS}_{\mathrm{a}}\end{array}$ & $\begin{array}{l}12.37 \\
* * * a b\end{array}$ & $\begin{array}{l}18.11 \\
{ }^{* *} \mathrm{abc}\end{array}$ & $\begin{array}{l}26.82 \\
* * * a b c d\end{array}$ & $\begin{array}{l}99.93 \\
* * * a b c,\end{array}$ & $\begin{array}{l}318.54 \\
* * * a b c d e f\end{array}$ & $\begin{array}{l}0.67 \\
\text { NSabcdefg }\end{array}$ \\
\hline 17 & 1.32 & $\begin{array}{l}34.38 \\
* * * a\end{array}$ & $\begin{array}{l}32.89 \\
* * * a N S b\end{array}$ & $\begin{array}{l}32.62 \\
* * * a^{N S} b c\end{array}$ & $\begin{array}{l}37.64 \\
* * * a^{N S} b c d\end{array}$ & $\begin{array}{l}175.92 \\
* * * a b c d e\end{array}$ & $\begin{array}{l}217.48 \\
* * a b c d e f\end{array}$ & $\begin{array}{l}0 \\
{ }^{N S} \\
a b c d e f g\end{array}$ \\
\hline 23 & 15.42 & $\begin{array}{l}15.42 \\
\mathrm{NS}_{\mathrm{a}}\end{array}$ & $\begin{array}{l}16.76 \\
\mathrm{NS} a b\end{array}$ & $\begin{array}{l}17.81 \\
\text { NSabc }\end{array}$ & $\begin{array}{l}33.87 \\
* a^{N S} b c d\end{array}$ & $\begin{array}{l}76.15 \\
* * * a b c d{ }^{* *} e\end{array}$ & $\begin{array}{l}158.39 \\
* a b c d e f\end{array}$ & $\begin{array}{l}0.32 \\
\text { NSabcdefg }\end{array}$ \\
\hline 24 & 2.42 & $\begin{array}{l}5.42 \\
\mathrm{NS}_{\mathrm{a}}\end{array}$ & $\begin{array}{l}7.69 \\
{ }^{N S} a b\end{array}$ & $\begin{array}{l}7.88 \\
\text { NSabc }\end{array}$ & $\begin{array}{l}12.7 \\
* * * a * b c d\end{array}$ & $\begin{array}{l}51.29 \\
* * * a b c d e\end{array}$ & $\begin{array}{l}71.32 \\
* * * a b c d e f\end{array}$ & $\begin{array}{l}0 \\
{ }^{N S} \\
a b c d e f g\end{array}$ \\
\hline 28 & 7.21 & $\begin{array}{l}7.21 \\
\text { Nsa }\end{array}$ & $\begin{array}{l}7.56 \\
{ }^{N S} \mathrm{ab}\end{array}$ & $\begin{array}{l}9.63 \\
\mathrm{NS}_{\mathrm{abc}}\end{array}$ & $\begin{array}{l}13.28 \\
\text { *abc }{ }^{N S} d\end{array}$ & $\begin{array}{l}109.35 \\
* * * a b c d e\end{array}$ & $\begin{array}{l}71.83 \\
* * * a b c d e f\end{array}$ & $\begin{array}{l}0.11 \\
\text { Nsabcdefg }\end{array}$ \\
\hline 35 & 1.94 & $\begin{array}{l}3.65 \\
\mathrm{Ns}_{\mathrm{a}}\end{array}$ & $\begin{array}{l}4.21 \\
\mathrm{NS}_{\mathrm{ab}}\end{array}$ & $\begin{array}{l}5.6 \\
{ }^{N S} \mathrm{abc}\end{array}$ & $\begin{array}{l}6.44 \\
\text { NS abcd }\end{array}$ & $\begin{array}{l}45.68 \\
* * * a b c d e\end{array}$ & $\begin{array}{l}52.09 \\
* * * a b c d e f\end{array}$ & $\begin{array}{l}0 \\
{ }^{N s} a b c d e f g\end{array}$ \\
\hline 37 & 1.25 & $\begin{array}{l}7.52 \\
* a\end{array}$ & $\begin{array}{l}7.86 \\
{ }^{*} a^{N S} b\end{array}$ & $\begin{array}{l}8.21 \\
{ }^{*} a^{N S} b c\end{array}$ & $\begin{array}{l}12.23 \\
* * * a * b c d\end{array}$ & $\begin{array}{l}45.73 \\
* * * a b c d e\end{array}$ & $\begin{array}{l}69.43 \\
* * * a b c d e f\end{array}$ & ${ }_{\mathrm{NS}}^{0}$ abcdefg \\
\hline 39 & 0.09 & $\begin{array}{l}2.11 \\
\mathrm{NS}_{\mathrm{a}}\end{array}$ & $\begin{array}{l}2.2 \\
{ }^{N S} a b\end{array}$ & $\begin{array}{l}2.45 \\
\mathrm{NS}_{\mathrm{abc}}\end{array}$ & $\begin{array}{l}3.05 \\
\text { NS abcd }\end{array}$ & $\begin{array}{l}28.97 \\
* * * a b c d e\end{array}$ & $\begin{array}{l}41.83 \\
* * * a b c d e f\end{array}$ & ${ }^{0}$ NSabcdefg \\
\hline 41 & 8.48 & $\begin{array}{l}22.15 \\
* * a\end{array}$ & $23.14{ }^{* *} a^{N S} b$ & $\begin{array}{l}26.71 \\
* a^{N S} \text { bc }\end{array}$ & $\begin{array}{l}26.24 \\
* * a^{N S} \text { bcd }\end{array}$ & $\begin{array}{l}149.25 \\
* * * a b c d e\end{array}$ & $\begin{array}{l}246.93 \\
* * * a b c d e f\end{array}$ & $\begin{array}{l}0 \\
{ }^{N S} \\
\text { abcdefg }\end{array}$ \\
\hline 49 & 11.28 & $\begin{array}{l}23.61 \\
* a\end{array}$ & $\begin{array}{l}23 \\
{ }^{*} a^{N S} b\end{array}$ & $\begin{array}{l}23.57 \\
{ }^{*} a^{N S} b c\end{array}$ & $\begin{array}{l}32.76 \\
* * a * b c d\end{array}$ & $\begin{array}{l}214.62 \\
* * * a b c d e\end{array}$ & $\begin{array}{l}341.59 \\
* * a b c d e f\end{array}$ & $\begin{array}{l}0.14 \\
\text { NSabcdefg }\end{array}$ \\
\hline 50 & 8.72 & $31.03 *{ }^{*} \mathrm{a}$ & $\begin{array}{l}31.5 \\
* * a{ }^{N S} b\end{array}$ & $\begin{array}{l}32.6 \\
* * a^{N S} \text { bc }\end{array}$ & $\begin{array}{l}33.02 \\
* a^{N S} \text { bcd }\end{array}$ & $\begin{array}{l}172.18 \\
* * * a b c d e\end{array}$ & $\begin{array}{l}216.61 \\
* * a b c d e f\end{array}$ & $\begin{array}{l}0 \\
\text { NS } a b c d e f g\end{array}$ \\
\hline
\end{tabular}

The results are expressed as mean $+\mathrm{SE}$ and $\mathrm{p}<0.05$ is considered significant. ${ }^{*} \mathrm{p}<0.05,{ }^{* *} \mathrm{p}<0.001,{ }^{* * *} \mathrm{p}<0.0001$

a-compared with control, b-compared with 0-5 years, T2DM, c-compared with 5-10 years T2DM, d-compared with 10-15 years T2DM, e-compared with

15-20 years T2DM, f-compared with microalbuminuria, g-compared with diabetic nephropathy

NS, non significant

aligned with Clustal Omega software. The calculation of molecular weight and pI of the probable protein was performed with ProtParam ExPASY tool. These 25 spots corresponded to total 13 different proteins. The details are given in Table 3. Post-translational modification were studied with GlycoMod and FindMod tools of ExPASY database.

\section{Two-dimensional urinary protein profile}

To avoid false positive estimation five patients with similar urinary 1 dimensional protein profile from each group were selected for further $2 \mathrm{DE}$ study. The $2 \mathrm{DE}$ of each selected participants were carried out twice. We found transthyretin/prealbumin and Ig kappa $C$ chain region in $0-5$ years of T2DM duration (Table 3, Fig. 2a). In T2DM patients with 5-10 years, we obtained transthyretin/prealbumin, Ig kappa $C$ chain region, Cystatin, $\mathrm{C}$, and Ubiquitin (Table 3, Fig. 2b). In 10-15 and 15-20 years of T2DM duration, we observed the appearance of Alpha 1 acid glycoprotein 1, Apolipoprotein A1, transthyretin/prealbumin, Ig kappa $C$ chain region, AMBP, Pigment epithelium-derived factor, Cystatin C, Zinc Alpha 2 glycoprotein and Ubiquitin (Table 3, Fig. 2c, d). Further, microalbuminuria and diabetic nephropathy patients marked the excretion of Ig kappa chain V-II, protein 25, Ig lambda 2 chain region, Alpha 1 acid glycoprotein 1, Apolipoprotein A1, transthyretin/prealbumin, Ig kappa $C$ chain region, AMBP, Cystatin $C$, zinc alpha 2 glycoprotein and Ubiquitin (Table 3, Fig. 2e, f). The above-mentioned proteins are significantly excreted in urine, whereas, serum creatinine, microalbumin, and eGFR were still within normal range. The proteins were involved in cell development, cell organization, defense response, metabolism, and signal transduction.

We have categorized the LMWPs into three different groups depending upon their excretion during the time interval of T2DM. Four proteinsincluded in Group 1 namely, Transthyretin/prealbumin, Ig kappa $C$ chain region, Cystatin $\mathrm{C}$ and Ubiquitin excreted in early years (0-5 years) of T2DM and significantly expressed till DN. Amongst which transthyretin and Ig kappa chains excreted as early as $0-5$ years of T2DM. However, transthyretin is not expressed after 10-15 years of T2DM. Ig kappa chains excretes through out the course of DN, right form 0 to 5 years T2DM up to DN. Cystatin c and Ubiquitin also excretes right form 0 to 5 years T2DM up to DN. From our study we can interpret that these four proteins either individually or in combination can prove 
a 2D Profile of 0 -5 yrs T2DM Group

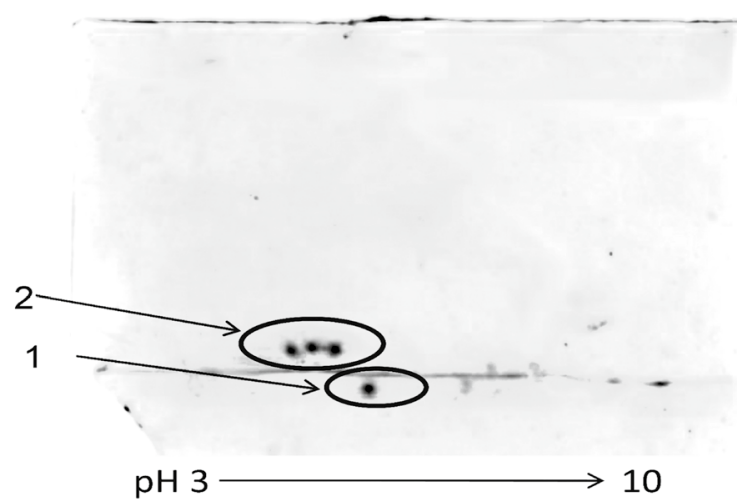

c 2D Profile of 10 -15 yrs T2DM Group

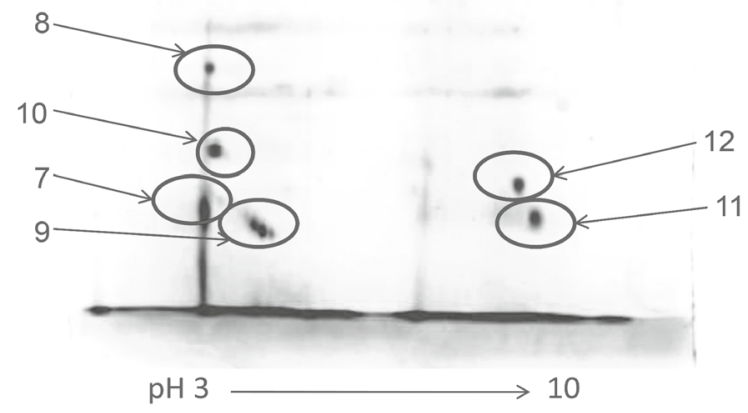

e 2D Profile of Microalbuminuria Group

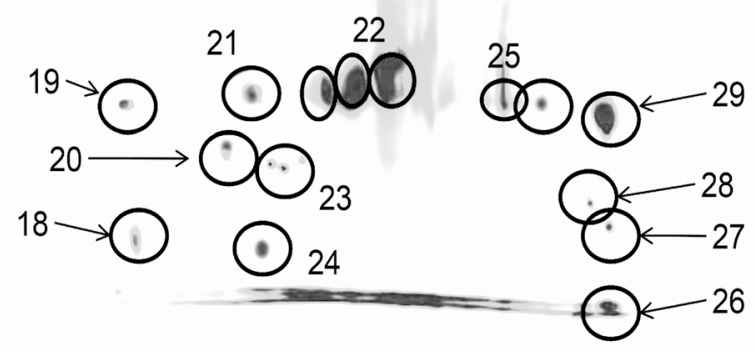

$\mathrm{pH} 3$

\section{b 2D Profile of 5-10 yrs T2DM Group}

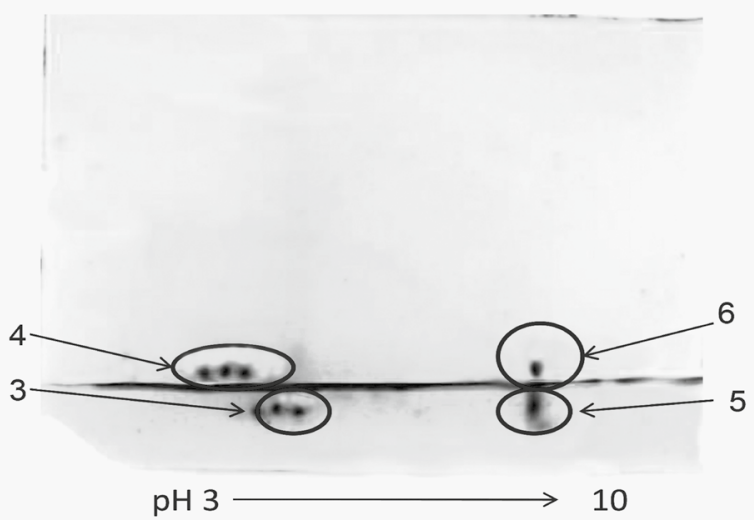

d 2D Profile of 15-20 yrs T2DM Group

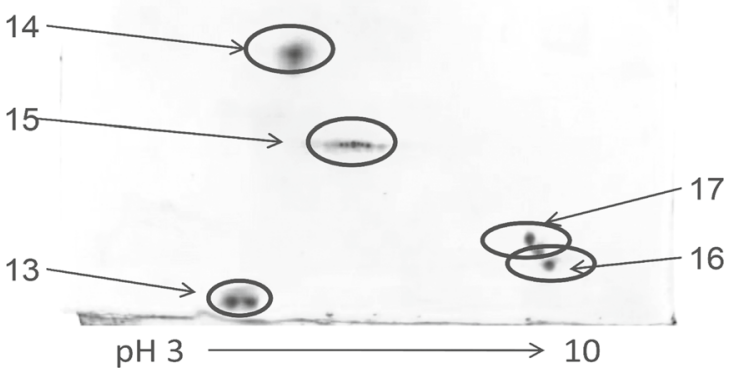

f 2D Profile of Diabetic Nephropathy Group

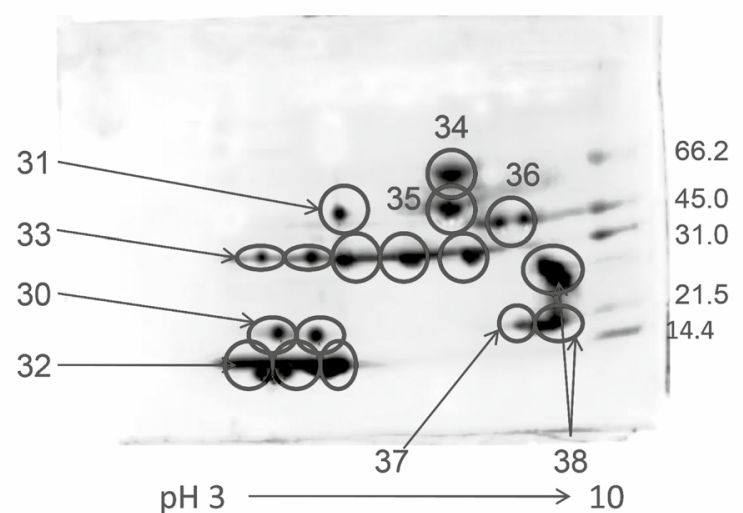

Fig. 22 dimensional urinary proteomic profile of various study groups. 2 dimensional proteome map of different study group. As the duration of T2DM increases the excretion of low molecular weight protein also increases. The proteins were identified with MALDI QTOF. Upregulation of the low molecular weight proteins can be observed as the participants progresses to macroalbuminuria

as potential low molecular weight candidates capable of diagnosing early diabetic nephropathy. Group 2 includes Alpha1 acid glycoprotein1, Apolipoprotein A1, Zinc alpha 2 glycoprotein, Alpha 1 Microglobulin/Bikunin
Precursor appear in late years of T2DM (at least in our patients) i.e. $10-15$ and $15-20$ years. Finally, Group 3 includes Apolipoprotein A IV, Alpha 1 antitrypsin, Haptoglobin, Ig kappa chain V-II, Protein 25, Ig lambda 2 
Table 3 Urinary proteins identified with MALDI-QTOF

\begin{tabular}{|c|c|c|c|c|c|c|}
\hline Spot no & Accession no & Protein name & $\mathrm{MW}(\mathrm{kDa})$ & $\mathrm{pl}$ & $\begin{array}{l}\text { Protein/peptide excreted } \\
\text { during }\end{array}$ & $\begin{array}{l}\text { Classification of LMWPs } \\
\text { depending upon its early } \\
\text { excretion }\end{array}$ \\
\hline $1,3,9$ & P02766 & Transthyretin/prealbumin & 15.88 & 5.69 & $\begin{array}{l}0-5,5-10 \text { and } 10-15 \text { years of } \\
\text { T2DM }\end{array}$ & \multirow{4}{*}{$\begin{array}{l}\text { Group 1: LMWPs excreting in } \\
\text { early years i.e. } 0-5 \text { and } 5-10 \\
\text { years T2DM }\end{array}$} \\
\hline $2,4,13,24,32$ & P01834 & Ig kappa C chain region & 16.60 & 5.58 & $\begin{array}{l}0-5,5-10,15-20 \text { years of T2DM, } \\
\text { MIA and DN }\end{array}$ & \\
\hline $5,11,16,26,27,37$ & P01034 & Cystatin C & 15.79 & 9.00 & $\begin{array}{l}\text { 5-10, 10-15, 15-20 years of } \\
\text { T2DM, MIA and DN }\end{array}$ & \\
\hline $6,12,17,28,29,38$ & P62979 & Ubiquitin & 17.96 & 9.00 & $\begin{array}{l}5-10,10-15,15-20 \text { years of } \\
\text { T2DM, MIA and DN }\end{array}$ & \\
\hline $7,8,18,19$ & P02763 & Alpha 1 acid glycoprotein 1 & 23.51 & 4.93 & 10-15 years of T2DM and MIA & \multirow{4}{*}{$\begin{array}{l}\text { Group 2: LMWPs excreting } \\
\text { in later years i.e. 10-15 and } \\
\text { 15-20 years T2DM }\end{array}$} \\
\hline 10 & P02647 & Apolipoprotein A1 & 30.77 & 5.56 & 10-15 years of T2DM & \\
\hline 14 & P25311 & Zinc alpha 2 glycoprotein & 37.25 & 5.71 & 15-20 years of T2DM & \\
\hline 15,33 & P02760 & $\begin{array}{l}\text { Alpha } 1 \text { microglobulin/ } \\
\text { bikunin precursor }\end{array}$ & 35.99 & 5.95 & $15-20$ years of T2DM and DN & \\
\hline 20,21 & P06727 & Apolipoprotein A IV & 45.39 & 5.28 & MIA & \multirow{6}{*}{$\begin{array}{l}\text { Group 3: LMWPs excreting in } \\
\text { advanced stages i.e. MIA } \\
\text { and DN }\end{array}$} \\
\hline $22,23,31$ & P01009 & Alpha 1 antitrypsin & 46.73 & 5.37 & MIA, DN & \\
\hline 25 & P00738 & Haptoglobin & 45.20 & 6.13 & MIA & \\
\hline 30 & P01614 & Ig kappa chain V-II & 12.67 & 5.28 & DN & \\
\hline 34,35 & Q86WR0 & Protein 25 & 24.47 & 6.34 & DN & \\
\hline 36 & P0CG05 & Ig lambda 2 chain region & 11.29 & 6.91 & DN & \\
\hline
\end{tabular}

Proteins were recognized using NCBI and SWISS PROT database of urine. The molecular weight and pl of the protein were calculated using ProtParam ExPASY tool

chain region appear after the appearance of microalbumin and hence they are over expressed in patients with MIA and DN.

\section{Discussion}

The most significant advantage of urinary proteomics is the prospect of a non-invasive and easy sampling system of diagnosis. Amongst the urinary proteome, $70 \%$ of the urinary proteins are of renal origin, and $30 \%$ are of systemic origin [4]. Thus, urinary proteomics may reduce the need for renal biopsy as the principal method for the diagnosis of the renal disorder. One of the major focus of our study was to establish a proteome map of low molecular weight proteinuria $<50 \mathrm{kDa}$ with the help of $2 \mathrm{DE}$ in T2DM patients with/without complications and varying duration as well as chronicity of diabetes.

From one-dimensional protein profiling, we were able to screen total 11 different LMWPs with molecular weight $13,17,23,24,28,35,37,39,41,49$ and $50 \mathrm{kDa}$ significantly predicting early DN. However, Visith et al. on multiple comparisons revealed nine spectra corresponding to 9 different individual bands (proteins ranging from 3.0 to $31.0 \mathrm{kDa}$ ) which significantly differentiated diabetic nephropathy from normal [8]. This variation may be due to the difference in sample size and study population. Our study includes four T2DM groups with varying duration of diabetes (ranging from 1 to 20 years) without any other secondary complications. T2DM patients with
0-5 years of diabetes duration showed protein profile analogous to that of the NHA group with an insignificant excretion of proteins. We can thus predict that the cutoff value of T2DM duration for the onset of renal alterations is about 5 years which resemble the observation reported by Rao et al. [9]. We for the first time reported a notable appearance of LMWPs $(<50 \mathrm{kDa})$ in the patients with 5-10 years of T2DM duration which can be attributed to protein overload or inefficiency of proximal tubules to reabsorb LMWPs [10]. We observed the presence of LMWPs in T2DM patients with normal renal functioning (serum creatinine and microalbuminuria still within normal ranges) indicating normal glomerular filtration and minimal proteinuria. This is indicative of tubular proteinuria/protein overload phase before the appearance of macroalbuminuria from where the condition can be reverted or ceased to worsen further.

There are many studies showing the utility of $2 \mathrm{DE}$ for establishing proteome map useful in differentiating DN patients from control groups [11-13]. But we for the first time screened excretion of proteins depending upon the duration of T2DM. From our observations, we found total eight LMWPs (Table 3; Group 1 and 2) capable of predicting the earliest subclinical renal alterations when serum creatinine, eGFR, and microalbuminuria are within normal range. From Group 1, Ig kappa light chains (KLC) showed their excretion as early as $0-5$ years of T2DM which even Groop et al. had 
reported that T2DM subjects (type 1 and type 2 both) with short duration (1 year) and normoalbuminuria excreted KLC [14]. It was found that nonenzymatic glycation of KLC interferes with its normal tubular reabsorption leading to its direct excretion in urine [14]. In addition to this Hutchison et al. has also reported fivefold urinary KLC in patients with progressive renal injury [15]. A study reported by Zurbig et al. showed the importance of collagen fragments in early detection of DN [16]. Another LMWP, i.e., transthyretin/prealbumin though excretes as early as $0-5$ years of T2DM but surprisingly it was not expressed after $10-15$ years of T2DM. However, this observation was not in accordance with Bellei et al. who found transthyretin to be progressively increase from T2DM patients with normoalbuminuria to DN [12], whereas, on the other hand, Rao et al. found it to be downregulating [11].

Excretion of cystatin c and ubiquitin was increased in patients with the duration of 5-10 years T2DM. We for the first time showed the expression of cystatin $\mathrm{c}$ in urine. Cystatin $\mathrm{c}$ is reabsorbed and catabolized in proximal tubules and hence its diagnostic excretion is increased in proximal tubular injury [17]. Dihazi et al. reported a shorter form of ubiquitin protein $(\approx 8 \mathrm{kDa})$ efficiently diagnosing DN subjects [18]. Oxidative and carbonyl stress regulates the excessive ubiquitin expression [19], whereas, its selective expression in the renal tubules suggests that the ubiquitin-proteasome proteolytic system is active in this compartment of the kidney and plays a vital role in the progression of $\mathrm{DN}$ [18]. But none of the study apart from us has mentioned the time-dependent appearance of LMWPs.

Hyperglycemia could lead to LMWPs excretion by affecting glomerular filtration and the tubular reabsorption of LMWPs. Christiansen et al. showed increased GFR due to moderate hyperglycemia in normal participants attained by intravenous glucose infusion [20]. In addition to this even glucose-induced osmotic diuresis could increase excretion of proximal tubule-derived LMWPs. There are several well known pathologic conditions related to proximal tubules responsible for the increase/decrease in LMWPs. The rate of protein filtration at the glomerulus and its reabsorption at proximal tubules might not be to the same extent. Thus, it depicts that the renal handling of the individual protein is completely different. The excreted urinary LMWPs can reveal the mechanism of its appearance or disappearance. Proteins/peptides directly appear in urine only if they have avoided tubular reabsorption and secreted by renal cells or lower urinary tract. The structural and functional proximal tubular changes during the early course of DN lead to the increased excretion of LMWPs $>40 \mathrm{kDa}[10]$. This implies that proximal tubular damage in T2DM subjects plays a vital role in progression to macroalbuminuria and increased LMWP excretion may imply renal tubular disorder in T2DM.

Jain et al. found 3 LMWPs namely Alpha 1 Microglobulin/Bikunin Precursor (A1M), Alpha1 acid glycoprotein1 (A1AG1) and Zinc alpha 2 glycoprotein (ZA2G) capable of discriminating diabetic nephropathy from control subjects but they could not recognize the precise stage of renal injury these LMWPs were excreted [21], whereas, we observed A1AG1, Apolipoprotein A1 (ApA1), ZA2G and $\mathrm{A} 1 \mathrm{M}$ appeared in late years of T2DM, i.e., $10-15$ and 15-20 years. A1AG1 is a well-established marker of inflammation and has been reported to predict mortality in T2DM subjects [22]. Even Rao et al. reported upregulation of ZA2G levels and it is the possible candidate for regulation body weight $[11,20]$. A1M levels were significantly correlated to the duration and progression of T2DM [23]. They reported that A1M levels were highest amongst the subjects on insulin treatment which correlated with our observations, where we found that subjects with 15-20 years of T2DM duration (late years of T2DM) also were dependent more on insulin treatment for controlling hyperglycemia $(\approx 30.8 \%$ subjects) in comparison to other T2DM patients w/o secondary complications.

Apolipoprotein A IV (Apo A4), Alpha 1 antitrypsin (A1AT), Haptoglobin (Hp), Ig kappa chain V-II/Ig lambda 2 chain (Ig chains) and Protein 25 appear in well-advanced stages. Apo A4 is significantly correlated with subjects having macular edema [24]. A1AT was first reported by Rao et al. to be upregulated [11] and Ig chains has a vital role in inflammation. Hp is a transport protein, and $\mathrm{Hp}$ allele 2 has a vital role in the development of DN [25]. The role of protein 25 is not well defined, but it is a predicted substrate of insulin receptor ad has a vital role in cell signaling. Its expression is ubiquitous in tissues and is released in urine due to tissue damage.

Megalin and cubulin are two endocytic receptors which both together facilitate reabsorption of almost all LMWPs, including albumin [26]. However, the affinity of RBP, $\beta-2$ microglobulin, NGAL and Vitamin D-binding protein to megalin is very low which indicates that their urinary excretion should increase along with the progression of albuminuria. Surprisingly we did not observe excretion of any above-mentioned such LMWPs in our study groups. These observations infer that an increase in urinary excretion of LMWPs is dependent on the degree to which the LMWPs compete for reabsorption in comparison to albumin. Even Groop et al. due to similar reason found increased urinary excretion of KLC (23 kDa) rather than $\beta-2$ microglobulin (12 kDa) [14]. Since, the LMWPs concentrations are affected by both competition for absorption and tubular injury [27]. Even, Thielemans 
et al. demonstrated the ability of albumin to inhibit reabsorption of certain LMWPs indicative of competition for a common transport mechanism [28]. Excretion of all the above-mentioned LMWPs indicates that their affinity for megalin-cubulin is decreased. Overall the urinary concentration of individual LMWP is dependent upon (1) filtration rate of LMWPs at the glomerulus, (2) its reabsorption rate at proximal tubule and (3) competition for absorption by albumin.

We also noted a difference in 2D patterns of urinary proteins in different groups of patients (Fig. 2). Variations in such 2D patterns are due to the difference in the physiological processes like glomerular filtration, tubular reabsorption of the proteins, blood pressure, renal blood flow, glomerular capillary pressure and blood levels of vasoactive peptides or hormones [13]. Interestingly, T2DM patients with normoalbuminuria gave a different protein pattern in comparison to T2DM patients with MIA and DN. The position of similar proteins like Transthyretin and IgG kappa chain $C$ region was shifted in the case of normoalbuminuric and macroalbuminuric T2DM patients. We observed that the molecular weight and pI varied from its theoretical molecular weight. Lafitte et al. also reported the similar difference between actual observed and theoretical molecular weight of protein [13]. This discrepancy might be due to post-translational modifications like glycosylation/phosphorylation [29]. An additional reason might be the low buffering capacity of small proteins, which are unable to migrate at their original isoelectric point [30].

Though acetone precipitation is one of the best technique for isolation of LMWPs, as it can recover the widest range of LMWPs from urine [31]. We observed that protein of molecular weight less than $11 \mathrm{kDa}$ were not isolated. This might be due to protein size plays a critical role in its precipitation by acetone, as the solvent concentration required for precipitation is inversely proportional to protein size [32]. Smaller proteins are more difficult to precipitate because their little-charged surface area produces less repulsive [32].

\section{Conclusion}

We have assessed urinary proteins with molecular weight $<50 \mathrm{kDa}$ by 2 -D gel electrophoresis and MALDI Q-TOF and found a small number of proteins that were upregulated at the different time interval of T2DM and their levels increased along with the increasing degree of albuminuria. These proteins were exclusive for T2DM and proteins with significant excretion in nondiabetic nephropathies were excluded from the study. This makes the current study unique amongst the past studies carried out till date. To determine the prospective efficacy of the candidate proteins we have included a well-defined and well-characterized patient population. The participants included are specifically age-matched, biochemical profile, matched and belonged to similar diabetes duration. Thus, this will not detract the limitation of the present experiment which is cross-sectional and with a restricted number of participants in different study groups. Regardless of this, a longitudinal study is required for validating the efficiency of the suggested proteomic pattern. Studying the patterns at regular interval for certain duration of time will help to infer the rate of progression of disease and closely monitor the patients for the same. We also accept the fact that the conventional methods like $\mathrm{HbA1C}$ and urinary dipstick methods help to monitor the disease and are by far the gold standard methods, but one cannot deny the fact that they are the markers of well established disease. The dipstick method can conventionally diagnose proteinuria at $300 \mathrm{mg} /$ day and $\mathrm{HbA1C}$ can predict well established diabetes. However, our present study can provide a better and early prediction to the insight of such slow progressive disease and can work better than $\mathrm{HbA} 1 \mathrm{C}$ and the traditional urinary dipstick method. The data finally suggested the utility of proteomic analysis be useful for detection of low abundant proteins. We have identified eight different proteins present in T2DM patients (with and without complications) which can be further characterized by ELISA or western blotting to confirm their utility in detecting early diabetic nephropathy. Out of which four proteins (Ig light chains, transthyretin, cystatin $\mathrm{c}$ and ubiquitin) either individually or in combination can prove as potential low molecular weight candidates capable of diagnosing early diabetic nephropathy as early as $0-5$ years of T2DM and 5-10 years of T2DM but their validation in large number of population is to be verified.

\section{Additional file}

Additional file 1. Urinary protein electeropherograms of control groups, participants with varrying duration of diabetes with normal kidney functioning, microalbuminuria and nephropathy.

\section{Authors' contributions}

KK conceived the idea, supervised the entire work and helped with rectification of manuscript. DN participated in the design of the study, performed the experiments and statistical analysis. Both authors read and approved the final manuscript.

\section{Author details}

${ }^{1}$ Department of Medical Laboratory Technology, Charotar Institute of Paramedical Sciences, CHARUSAT, CHARUSAT-Campus, Highway 139, Off, Nadiad-Petlad Road, Changa, Gujarat 388421, India. ${ }^{2}$ Present Address: National Institute of Pharmaceutical Education and Research (NIPER-G), Gandhinagar Opposite Air Force Station, Palaj, Gandhinagar, Gujarat 382355, India. 


\section{Acknowledgements}

The authors thank Head and staff members, Animal Biotechnology department, Anand Agricultural University, Anand, Gujarat for providing technical help with Bioanalyzer. We also thank SAIF, IIT Mumbai, Mumbai, Maharashtra for extending their help in MALDI-QTOF analysis. The authors in addition thank the physicians and staff of Muljibhai Patel Urological Hospital, Nadiad, Gujarat, for screening of patients.

\section{Competing interests}

The authors declare that they have no competing interests.

\section{Availability of data and materials}

Data are all contained within the paper.

\section{Consent for publication}

Not applicable.

\section{Ethics approval and consent to participate}

All procedures followed were in accordance with the ethical standards of the Ethical Committee of Muljibhai Patel Urological Hospital.

\section{Funding}

The Microfluidic array was carried out with the fund provided by student research fund of Bioscience Department, Sardar Patel University. The MALDI QTOF analysis was funded by National Institute of Pharmaceutics Education and Research-Gandhinagar.

\section{Publisher's Note}

Springer Nature remains neutral with regard to jurisdictional claims in published maps and institutional affiliations.

Received: 24 January 2019 Accepted: 20 April 2019

Published online: 17 May 2019

\section{References}

1. Riaz S. Diabetes mellitus. Sci Res Essay. 2009;4:367-73.

2. Caramori ML, Fioretto P, Mauer M. The need for early predictors of diabetic nephropathy risk: is albumin excretion rate sufficient? Diabetes. 2000;49:1399-408.

3. Thongboonkerd V. Proteomics in nephrology: current status and future directions. Am J Nephrol. 2004;24:360-78.

4. Thongboonkerd V, Malasit P. Renal and urinary proteomics: current applications and challenges. Proteomics. 2005;5:1033-42.

5. Kroll MH, Chesler R, Hagengruber C, Blank DW, Kestner J, Rawe M. Automated determination of urinary creatinine without sample dilution: theory and practice. Clin Chem. 1986;32(3):446-52.

6. Cockroft DW, Gault MH. Prediction of creatinine clearance from serum creatinine. Nephron. 1976;16:31-41.

7. Parker KM, England JD, Costa J, Hess RL, Gloldstein DE. Improved colorimetric assay for glycated hemoglobin. Clin Chem. 1981;27(5):669-72.

8. Thongboonkerd V, Songtawee N, Sritippayawan S. Urinary proteome profiling using microfluidic technology on a chip. J Proteome Res. 2007;6:2011-8.

9. Rao X, Wan M, Qiu C, Jiang C. Role of cystatin C in renal damage and the optimum cut-off point of renal damage among patients with type 2 diabetes mellitus. Exp Ther Med. 2014;8:887-92.

10. Barratt J, Topham P. Urine proteomics: the present and future of measuring urinary protein components in disease. CMAJ. 2007;177:361-8.

11. Rao PV, Lu X, Standley M, Pattee P, Neelima G, Girisesh G, Dakshinamurthy K, Roberts CT Jr, Nagalla SR. Proteomic identification of urinary biomarkers of diabetic nephropathy. Diabetes Care. 2007;30:629-37.
12. Bellei E, Rossi E, Lucchi L, Uggeri S, Albertazzi A, Tomasi A, lannone A. Proteomic analysis of early urinary biomarkers of renal changes in type 2 diabetic patients. Proteomics Clin Appl. 2008;2:478-91.

13. Lafitte $D$, Dussol B, Andersen $S$, Vazi A, Dupuy $P$, Jensen $O N$, Berland $Y$, Verdier JM. Optimized preparation of urine samples for two-dimensional electrophoresis and initial application to patient samples. Clin Biochem. 2002;35:581-9.

14. Groop L, Makipernaa A, Stenman S, Defronzo RA, Teppo AM. Urinary excretion of kappa light chains in patients with diabetes mellitus. Kidney Int. 1990;37:1120-5

15. Hutchison CA, Harding S, Hewins P, Mead G, Townsend J, Bradwell AR, Cockwell P. Quantitative assessment of serum and urinary polyclonal free light chains in patients with chronic kidney disease. Clin J Am Soc Nephrol. 2008:3:1684-90.

16. Zürbig P, Jerums G, Hovind P, Maclsaac R, Mischak H, Nielsen Panagiotopoulos S, Persson F, Rossing P. Urinary proteomics for early diagnosis in diabetic nephropathy. Diabetes. 2012;61:3304-13.

17. Kaseda R, lino N, Hosojima M, Takeda T, Hosaka K, Kobayashi A, Yamamoto K, Suzuki A, Kasai A, Suzuki Y, Gejyo F, Saito A. Megalin-mediated endocytosis of cystatin C in proximal tubule cells. Biochem Biophys Res Commun. 2007;357:1130-4.

18. Dihazi H, Muller GA, Lindner S, Meyer M, Asif AR, Oellerich M, Strutz F. Characterization of diabetic nephropathy by urinary proteomic analysis: identification of a processed ubiquitin form as a differentially excreted protein in diabetic nephropathy patients. Clin Chem. 2007;53(9):1636-45.

19. Sun L, Pan X, Wada J, Haas CS, Wuthrich RP, Danesh FR, Chugh SS, Kanwar YS. Isolation and functional analysis of mouse UbA52 gene and its relevance to diabetic nephropathy. J Biol Chem. 2002;277:29953-62.

20. Christiansen SJ, Frandsen M, Parving H-H. Effect of intravenous glucose infusion on renal function in normal man and insulin-dependent diabetics. Diabetologia. 1981;21:368-73.

21. Jain S, Rajput A, Kumar Y, Uppuluri N, Arvind AS, Tatu U. Proteomic analysis of urinary protein markers for accurate prediction of diabetic kidney disorder. J Assoc Physicians India. 2005;53:513-20.

22. Christiansen MS, Hommel E, Magid E, Feldt-Rasmussen B. Orosomucoid in urine predicts cardiovascular and over-all mortality in patients with type II diabetes. Diabetologia. 2002;45:115-20.

23. Hong CY, Hughes K, Chia KS, Ng V, Ling SL. Urinary alpha 1-microglobulin as a marker of nephropathy in type 2 diabetic Asian subjects in Singapore. Diabetes Care. 2003;26:338-42.

24. Ouchi M, West K, Crabb JW, Kinoshita S, Kamei M. Proteomic analysis of vitreous from diabetic macular edema. Exp Eye Res. 2005;81:176-82.

25. Nakhoul F, Zoabi R, Kanter Y, Zoabi M, Skorecki K, Hochberg I, Leibu R, Miller B, Levy AP. Haptoglobin phenotype and diabetic nephropathy. Diabetologia. 2001;44:602-4.

26. Christensen I, Birn H. Megalin and cubilin: multifunctional endocytic receptors. Mol Cell Bio. 2002;3:258-68.

27. Nejat M, Hill JV, Pickering JW, Edelstein CL, Devarajan P, Endre ZH. Albuminuria increases cystatin $C$ excretion: implications for urinary biomarkers. Nephrol Dial. 2012;27(3):96-103.

28. Thielemans N, Lauwerys R, Bernard A. Competition between albumin and low-molecular-weight proteins for renal tubular uptake in experimental nephropathies. Nephron. 1994:66:453-8.

29. Sanchez LM, Chirino AJ, Bjorkman PJ. Crystal structure of human ZAG, a fat-depleting factor related to MHC molecules. Science. 1999;283:1914-9.

30. Wilkins MR, Gooley AA. Protein identification in proteome projects. Proteome Research: new frontiers in functional genomics. Berlin: Springer; 1997. p. 35-64.

31. Thongboonkerd V, Mcleish K, Arthur J, Klein J. Proteomic analysis of normal human urinary proteins isolated by acetone precipitation or ultracentrifugation. Kidney Int. 2002;62:1461-9.

32. Scopes RK, editor. Protein purification: principles and practice (3rd, ed). Heidelberg: Springer; 1994. 